\title{
Response functions and susceptibilities for multiresonant non-linear optical spectroscopy: perturbative computer algebra solution including feeding
}

\author{
Bernhard Dick ${ }^{1}$ \\ Max-Planck-Institut für biophysikalsche Chemie, Abtellung Laserphysik, W-3400 Göttingen, Germany
}

Received 18 August 1992

\begin{abstract}
A diagrammatic perturbation theory is presented which permits the calculation of a molecular non-linear optical response function for a particular time sequence of light pulses, or a molecular non-linear-optical susceptibility for a particular combination of frequencies of the input light fields. Molecular relaxation is treated in the Markov approximation. The method also accounts for incoherent population transfer between molecular levels as long as a hierarchical level ordering is possible. The diagrams are especially useful for the selective generation of terms containing a particular resonance of interest, or obeying the rotating wave approximation. The algorithm has been implemented in the symbulic algebra language REDUCE. These procedures allow the automatic generation of all diagrams evolving from a particular experimental situation, and the automatic calculation of the corresponding response function or susceptibility.
\end{abstract}

\section{Introduction}

In non-linear optical spectroscopic experiments a sample is irradiated with one or several laser light fields. Each of these can be characterized by a time-dependent amplitude and a frequency. (The frequency may also vary with time, corresponding to a chirped light pulse.) Through interaction with the laser fields a non-linear polarization $\boldsymbol{P}(\boldsymbol{r}, t)$ is generated in the sample which radiates new light fields. They can be calculated from Maxwell's inhomogeneous wave equation with the polarization $\boldsymbol{P}(\boldsymbol{r}, t)$ as source term $[1,2]$. Depending on the phase rclationship with the ingoing fields the new fields cause absorption and coherent or incoherent emission. Experiments are commonly distinguished as frequency-domain or time-domain experiments depending on whether the frequency or the time intervals of the ingoing and detected light pulses are varied as parameters. If phase and amplitude could be measured completely, both types of experiments would yield the same information. In praxis, however, this is not possible. Results of both types of experiments hence complement each other, and spectroscopy in both domains is useful.

In the traditional theoretical treatment of non-linear optical spectroscopy the optical polarization is expanded in powers of the field strength of the incident light beams which arc modclled as monochromatic plane waves. The response of the material in each order of the expansion is then given by a coupling constant of the material called its $n$ th-order non-linear optical susceptibility $\chi^{(n)}$. The use of monochromatic waves corresponds to the idealized situation of a frequency-domain experiment. It also implies the consideration of a steady state. Strictly speaking, therefore, susceptibilities apply only to molecular systems in thermal equilibrium. Time-domain experiments can also be discussed in the framework of susceptibilities. However, a more natural description for time domain experiments is provided by the molecular response functions $\mathbf{R}^{(n)}$. For a system in thermal equilibrium the susceptibilities and response functions are related by a Fourier transformation [2]. In addition, gen-

1 New address: Lehrstuhl für Chemie III, Institut für Physikalische und Theoretische Chemie, Universität Regensburg, W-8400 Regensburg, Germany. 
eralized response functions can be defined which also apply to non-equilibrium situations.

The macroscopic optical polarization is the ensemble average of the time-dependent induced dipole moments of the molecules in the irradiated volume. These can be obtained from a calculation of the temporal evolution of the molecular system under the influence of the external fields. This is normally done with time-dependent perturbation theory which yields the wavefunction or the statistical operator of the molecular system as a power series in the external field strengths [1]. Comparison with the series expansion of the optical polarization shows that each order of perturbation theory yields the susceptibility of the corresponding order. Thus measurable spectroscopic quantities like line positions, linewidths, and intensities can be connected to molecular properties like level energies, relaxation parameters, and transition dipoles.

The series expansion of the polarization is in most cases a good approximation. It can, however, break down if any of the ingoing fields is "strong" and its frequency comes close to resonance with an optically allowed molecular transition. Methods have been developed which, in certain situations, allow the treatment of these fields in a non-perturbative way [3-5]. For all situations considered in this paper we assume that the series expansion is applicable.

Time-dependent perturbation theory with a light field containing several Fourier components (i.e. colors) results in many terms. All possible time-orderings of these Fourier components for a propagation of the wavefunction or its complex conjugate must be considered. E.g., the general expression for a third order susceptibility has 48 terms [6]. The use of perturbation theory quasi-naturally suggests a diagrammatic technique. Early approaches described the temporal evolution of the wavefunction in close analogy to Feynman diagrams [7,8] but were only applicable to pure states. Systems with dephasing (coherence loss) are properly described by the statistical operator instead of the wavefunction. The corresponding diagrams must account for the propagation of its bra- and ket-part and their relative time ordering. This lead to the invention of double-sided Feynman diagrams consisting of two strings, one representing the bra, the other the ket [9-13]. An equivalent notation used a circle where each half corresponds to one of the strings [14-16]. The mathematical treatment lead to propagation rules, in which each radiative propagation step contributes a multiplicative factor to the susceptibility. Still another way of representing a particular contribution is by indicating the path in Liouville space along which the term is generated through successive evolutions on the bra or ket side. These diagrams are widely used by Mukamel and co-workers, e.g. in refs. [17-19]. We use similar evolution trees to enumerate all relevant paths (see ref. [20] and fig. 3).

These approaches did account for phase relaxation, but not for the possibility of incoherent population transfer between molecular levels, called feeding. For non-resonant situations this should be of little importance, but may have drastic effects with resonances. An attempt of including feeding into the diagrammatic technique has been published [21]. However, it starts from a transformation to a time-independent formulation of the Liouville equation [4] which is possible if only a single Fourier component need be considered for each matrix element of the statistical operator. This imposes the following restrictions: (i) Only one field may be resonant with a molecular level pair. (ii) Not more than $n-1$ fields are allowed for an $n$-level system. (iii) The rotating wave approximation (RWA) must be applicable. The present article describes a method that incorporates feeding into the diagrammatic perturbation theory without these restrictions. The result is a new propagation rule yielding a multiplicative factor to the susceptibility from each feeding step in the diagram.

A second new aspect of the present paper is the extension of the diagrammatic approach to the direct calculation of molecular response functions ${ }^{\# 1}$. They give the response of the system to a sequence of infinitesimally short light pulses. Each radiative propagation step yields a multiplicative factor as in the case of susceptibilities. Feeding can also be included and results in a substitution rule.

This paper bears to some extent review character since the diagrammatic technique for the calculation of susceptibilities in the absence of feeding has been described in several previous papers. However, I feel that it is justified to present the theory with its new aspects (feeding and response functions) in a comprehensive context.

"1 This possibility was briefly mentioned already in ref. [22]. 
In particular so since it also uses a different graphical representation of the diagrams which is based on the molecular level structure. Earlier diagrams of this kind did not distinguish between propagation of the bra- and the ket-part of the density operator and were thus ambiguous. Our diagrams, previously used in refs. [20,22], do not suffer from this restriction, but can be unambiguously mapped to the double-sided Feynman diagrams. The extension to include feeding is readily made in an intuitively comprehensive way. In addition, these diagrams allow an easy visualization of the leading terms in the rotating wave approximation or displaying particular molecular resonances. Thus the relevant terms can be selected directly from inspection of the diagram, and not by inspection of the formula that it generates. With respect to this the other types of diagrams are rather abstract.

Finally, we describe an implementation of the algorithm presented in this paper into the computer-algebra language REDUCE (for the newest version see ref. [23]). We use this system for almost two years now to generate all valid diagrams for a particular experimental situation, and automatically calculate the corresponding susceptibility and/or response function.

\section{Response functions and susceptibilities}

In order to understand the results of a nonlinear optical experiment on a molecular level, the macroscopic optical polarization $\boldsymbol{P}(\boldsymbol{r}, t)$ must be expressed in terms of molecular properties, in particular the energy level structure, the magnitude and direction of transition dipoles, and relaxation parameters. The quantum mechanical connection between the microscopic and the macroscopic quantities is given by

$\boldsymbol{P}(\boldsymbol{r}, t)=N \operatorname{Tr}\{\rho(\boldsymbol{r}, t) \boldsymbol{\mu}\}$,

where $\rho(\boldsymbol{r}, t)$ is the statistical operator (often called density matrix) of the molecular ensemble, and $N$ is the number density of molecules. This equation is valid for arbitrarily strong light fields. In many cases, however, it is possible to expand the polarization in a Taylor series in powers of the field strength,

$\boldsymbol{P}(t)=\boldsymbol{P}^{(0)}(t)+\boldsymbol{P}^{(1)}(t)+\boldsymbol{P}^{(2)}(t)+\boldsymbol{P}^{(3)}(t)+\ldots$.

This corresponds to a similar expansion of the statistical operator. In the following we assume that the response of the sample is local, i.e. the non-linear polarization at a particular point $r$ in space depends only on the light field at this point. Also, we consider a non-magnetic medium and represent the light beam by its electric field vector $\boldsymbol{E}(\boldsymbol{r}, t)$. When the Taylor series (eq. (2)) is valid, the most general relation between the non-linear polarization of a particular order and the electric field strength is given by the convolution

$\boldsymbol{P}^{(n)}(t)=\int \mathrm{d} t_{n} \ldots \int \mathrm{d} t_{1} \mathbf{Q}^{(n)}\left(t ; t_{n}, \ldots, t_{1}\right) \vdots \boldsymbol{E}\left(t_{n}\right) \ldots \boldsymbol{E}\left(t_{1}\right)$,

where $\mathbf{Q}^{(n)}\left(t ; t_{n}, \ldots, t_{1}\right)$ is a generalized response function. Causality requires that $\mathbf{Q}^{(n)}$ vanishes if any of the time arguments $t_{t}$ is larger than $t$. For a system in equilibrium this generalized response function is invariant against a shift of all time arguments by a constant time interval $\tau$. A function $\mathbf{R}^{(n)}$ can then be defined with one time argument less,

$\mathbf{Q}^{(n)}\left(t ; t_{n}, \ldots, t_{1}\right)=\mathbf{Q}^{(n)}\left(t-\tau ; t_{n}-\tau, \ldots, t_{1}-\tau\right)=\mathbf{Q}^{(n)}\left(0 ; t_{n}-t, \ldots, t_{1}-t\right)=\mathbf{R}^{(n)}\left(t_{n}-t, \ldots, t_{1}-t\right)$.

The causality condition implies that $\mathbf{R}^{(n)}$ is non-vanishing only for negative time arguments. This function is ordinarily called the response function. After introducing the Fourier transform ${ }^{\# 2}$ of the electric field,

\#2 This form is found in mathematical textbooks as well as in some books on non-linear optics [24-27]. With this definition an electric field component $\mathscr{E} \exp (\mathrm{i} \omega t)$ has positive frequency. Other books on non-linear optics use the opposite sign convention for $\omega$, e.g. [ 1,2 , 28,30 ], or put the factor $2 \pi$ into the integral over time [2]. The present choice is consistent with our previous usage of frequency arguments in the susceptibilities $[4,20,22]$. 
$E(\omega)=\int \mathrm{d} t \boldsymbol{E}(t) \exp (-\mathrm{i} \omega t)$

$\boldsymbol{E}(t)=\int \frac{\mathrm{d} \omega}{2 \pi} \boldsymbol{E}(\omega) \exp (\mathrm{i} \omega t)$

the definition of the non-linear polarization eq. (3) for the equilibrium system can be rewritten as

$\boldsymbol{P}^{(n)}(t)=\int \frac{\mathrm{d} \omega_{n}}{2 \pi} \ldots \int \frac{\mathrm{d} \omega_{1}}{2 \pi} \chi^{(n)}\left(\omega_{n}, \ldots, \omega_{1}\right) \vdots \boldsymbol{E}\left(\omega_{n}\right) \ldots \boldsymbol{E}\left(\omega_{1}\right) \exp \left(\mathrm{i} t \sum_{k=1}^{n} \omega_{k}\right)$,

where the non-linear susceptibility $\chi^{(n)}$ and the response function are related by

$\chi^{(n)}\left(\omega_{n}, \ldots, \omega_{1}\right)=\int \mathrm{d} t_{n} \ldots \int \mathrm{d} t_{1} \mathbf{R}^{(n)}\left(t_{n}, \ldots, t_{1}\right) \exp \left(\mathrm{i} \sum_{k=1}^{n} \omega_{k} t_{k}\right)$,
$\mathbf{R}^{(n)}\left(t_{n}, \ldots, t_{1}\right)=\int \frac{\mathrm{d} \omega_{n}}{2 \pi} \ldots \int \frac{\mathrm{d} \omega_{1}}{2 \pi} \chi^{(n)}\left(\omega_{n}, \ldots, \omega_{1}\right) \exp \left(-\mathrm{i} \sum_{k=1}^{n} \omega_{k} t_{k}\right)$.

For the present purpose it is sufficient to represent the incident light beams by a superposition of plane waves,

$$
\begin{aligned}
& \boldsymbol{E}(\boldsymbol{r}, t)=\frac{1}{2} \sum_{J}\left[\mathscr{E}_{J}(\boldsymbol{r}, t)+\mathscr{E}_{j}^{*}(\boldsymbol{r}, t)\right], \\
& \mathscr{E}_{J}(\boldsymbol{r}, t)=\boldsymbol{e}_{J} \mathscr{E}_{j}(t) \exp \left[\mathrm{i}\left(\omega_{j} t-\boldsymbol{k}_{j} \cdot \boldsymbol{r}\right)\right] .
\end{aligned}
$$

Each particular wave is characterized by its frequency $\omega_{j}$, its wavevector $\boldsymbol{k}_{j}$, and its amplitude $\mathscr{E}_{j}(t)$ which may be time-dependent. Eqs. (1) and (2) contain $r$ only as a parameter. The factors $\exp \left(-\mathrm{i} \boldsymbol{k}_{\boldsymbol{j}} \cdot \boldsymbol{r}\right)$ in eq. (10) hence appear only as multiplicative factors in $\boldsymbol{P}(\boldsymbol{r}, t)$. After solution of the wave equation this results in the well known phase matching conditions for parametric processes. For the discussion of the local response we may consider a molecule at the origin of the coordinate system and set $r=0$.

Let us now consider two ideal experimental situations. In the ideal frequency domain experiment the laser fields are monochromatic and their Fourier transform is a sum of $\delta$-functions,

$\boldsymbol{E}(\boldsymbol{r}, \omega)=\pi \sum_{J}\left[\mathscr{E}_{j} \delta\left(\omega_{J}-\omega\right) \exp \left(-\mathrm{i} \boldsymbol{k}_{j} \cdot \boldsymbol{r}\right)+\mathscr{E}_{J}^{*} \delta\left(\omega_{J}+\omega\right) \exp \left(\mathrm{i} \boldsymbol{k}_{j} \cdot \boldsymbol{r}\right)\right]$

The polarization calculated according to eq. (7) decomposes into a sum of monochromatic plane waves:

$$
\begin{aligned}
& \boldsymbol{P}(\boldsymbol{r}, t)=\frac{1}{2} \sum_{l}\left[\mathscr{P}_{l}(\boldsymbol{r}, t)+\mathscr{P}_{l}^{*}(\boldsymbol{r}, t)\right], \\
& \mathscr{P}_{l}(\boldsymbol{r}, t)=\boldsymbol{e}_{l} \mathscr{R}_{l} \exp \left[\mathrm{i}\left(\omega_{l} t-\boldsymbol{k}_{l} \cdot \boldsymbol{r}\right)\right] .
\end{aligned}
$$

For example, the third order polarization wave at frequency $\omega_{4}=\omega_{1}-\omega_{2}+\omega_{3}$ with wave vector $\boldsymbol{k}_{P}=\boldsymbol{k}_{1}-\boldsymbol{k}_{2}+\boldsymbol{k}_{3}$ is

$$
\mathscr{P}^{(3)}(\boldsymbol{r}, t)=\frac{3}{2} \chi^{(3)}\left(\omega_{1},-\omega_{2}, \omega_{3}\right): \mathscr{E}_{1} \mathscr{E}_{2}^{*} \mathscr{E}_{3} \exp \left[\mathrm{i}\left(\omega_{4} t-\boldsymbol{k}_{P} \cdot \boldsymbol{r}\right)\right]
$$

The numerical factor $\frac{3}{2}$ occurs since in the product $\boldsymbol{E E E}$ the term $\mathscr{E}_{1} \mathscr{E}_{2}^{*} \mathscr{E}_{3}$ appears six times. In the general case the numerical factor will be $N_{\mathrm{P}} / 2^{n-1}$, where $N_{\mathrm{P}}$ is the number of permutations of the electric field amplitudes, and $n$ is the order of the polarization. We obtain the result that the response of the molecular system in an ideal frequency-domain experiment is given by the susceptibility at the corresponding frequencies. It can hence be calculated from the molecular parameters in two steps. First the statistical operator of the molecular system under the influence of the monochromatic plane waves must be calculated. Then the polarization is obtained from eq. (1) and compared to the corresponding macroscopic expression, e.g. eq. (15).

The other idealized experimental situation considers the response of the molecular system when it is irradi- 
ated by a sequence of ultrashort light pulses. In this case the electric field can be written in the form

$\boldsymbol{E}(\boldsymbol{r}, t)=\frac{1}{2} \sum_{J}\left\{\mathscr{E}_{j} \exp \left[\mathrm{i}\left(\omega_{J} t-\boldsymbol{k}_{J} \cdot \boldsymbol{r}\right)\right]+\mathscr{E}_{J}^{*} \exp \left[-\mathrm{i}\left(\omega_{j} t-\boldsymbol{k}_{J} \cdot \boldsymbol{r}\right)\right]\right\} \Delta\left(t-\tau_{j}\right)$.

This ansatz characterizes each light pulse by a complex amplitude vector $\mathscr{E}_{j}$, a carrier frequency $\omega_{j}$, a wave vector $\boldsymbol{k}_{j}$ and a real temporal envelope function $\Delta(t)$ centered at time $\tau_{j}$. The notion of a carrier frequency is only valid if the temporal width of the pulse envelope is larger than the inverse frequency. This is still true for light pulses in the femtosecond range. In the limiting case of infinitely short light pulses the envelope function approaches the $\delta$-function and the carrier frequency loses its physical meaning.

The polarization $\boldsymbol{P}(\boldsymbol{r}, t)$ generated by the field of eq. (16) can be calculated with eq. ( 3 ) and is a sum of terms each corresponding to a particular pulse sequence in $\boldsymbol{E}(\boldsymbol{r}, t)$, a particular carrier frequency, and a wave vector,

$\boldsymbol{P}(\boldsymbol{r}, t)=\frac{1}{2} \sum_{l}\left\{\mathscr{P}_{l}(t) \exp \left[\mathrm{i}\left(\omega_{l} t-\boldsymbol{k}_{l} \cdot \boldsymbol{r}\right)\right]+\mathscr{P}_{l}^{*}(t) \exp \left[-\mathrm{i}\left(\omega_{l} t-\boldsymbol{k}_{l} \cdot \boldsymbol{r}\right)\right]\right\}$

The complex envelope functions $\mathscr{P}_{l}(t)$ reflect the molecular relaxation dynamics and will in general retain finite temporal widths even in the limit of infinitesimally short light pulses. As an example, let us consider a sequence of three light pulses with different frequencies $\omega_{1}, \omega_{2}$, and $\omega_{3}$. One of the terms in eq. (17) then describes a wave with carrier frequency $\omega_{4}=\omega_{1}-\omega_{2}+\omega_{3}$ and with wave vector $\boldsymbol{k}_{P}=\boldsymbol{k}_{1}-\boldsymbol{k}_{2}+\boldsymbol{k}_{3}$. Its amplitude in the $\delta$-pulse limit is the sum of the three terms containing the product $\mathscr{E}_{1} \mathscr{E}_{2}^{*} \mathscr{E}_{3}$ of field amplitudes:

$\mathscr{P}_{4}^{(3)}(t)=\frac{3}{2} \mathbf{Q}^{(3)}\left(t ; \tau_{1}, \tau_{2}, \tau_{3}\right): \mathscr{E}_{1} \mathscr{E}_{2}^{*} \mathscr{E}_{3} \exp \left[\mathrm{i}\left(\omega_{1} \tau_{1}-\omega_{2} \tau_{2}+\omega_{3} \tau_{3}\right)\right]$

In a realistic experiment the signal due to this process can be readily distinguished from that of other pulse sequences and other carrier frequencies through an appropriate choice of phase matching conditions. If the statistical operator is calculated for an idealized sequence of $\delta$-function pulses, a comparison of the polarization according to eq. (1) with the macroscopic expression, e.g. eq. (18), will yield the contribution of this particular pulse sequence to the response function in terms of molecular properties. A response function defined in this way corresponds to a single time sequence of pulses. Since the susceptibility describes the answer of the system in the steady state, it contains information about all possible time orderings of the input field components.

The fact that only one time sequence is considered in each term of the response function and only one frequency component in each term of the susceptibility is not a shortcoming but an advantage. Also in an actual experiment one is not interested in the complete response function or susceptibility, but only in such a particular contribution. The choice of phase matching conditions effectively selects a Fourier component of the signal to be measured, and the time delays between pulses can also be set by the experimenter.

\section{Perturbative calculation of the statistical operator}

In quantum mechanics the properties of a partially coherent molecular ensemble are completely described by its statistical operator $\rho$. Any macroscopic quantity is calculated as the trace of the product of the corresponding microscopic operator with $\rho$. Thus the optical polarization $\boldsymbol{P}(t)$ is the average of the microscopic dipole moment $\boldsymbol{\mu}$, as expressed in eq. (1). Typically the calculation is done in the basis of the eigenstates of the unperturbed Hamiltonian and the trace is written as a double sum,

$\boldsymbol{P}(t)=N \sum_{k l} \rho_{k l}(l) \boldsymbol{\mu}_{l k}$,

where $\rho_{k l}(t)=\langle k|\rho(t)| l\rangle$ are the matrix elements. The diagonal elements $\rho_{k k}(t)$ give the population of the molecular levels, whereas the off-diagonal elements $\rho_{k l}(t)$ are frequently called coherences since they are mainly responsible for the radiation of parametric signals. The temporal evolution of the statistical operator is determined by the Liouville equation, which in the interaction picture reads 
$\dot{\rho}=\frac{\mathrm{i}}{\hbar}[\rho, V]-(\dot{\rho})^{\mathbf{R}}$.

In the following we use the interaction operator between the molecules and the light fields in the dipole approximation, $V(t)=-\mu \cdot E(t)$. The operator $(\dot{\rho})^{\mathbf{R}}$ describes the relaxation of the system towards equilibrium in the absence of the external fields. In the Markov approximation its matrix elements are

$$
\begin{aligned}
& (\dot{\rho})_{k l}^{\mathrm{R}}=-\Gamma_{k l} \rho_{k l}, \\
& (\dot{\rho})_{k k}^{\mathrm{R}}=-\Gamma_{k k} \rho_{k k}+\sum_{m \neq k} \gamma_{m k} \rho_{m m} .
\end{aligned}
$$

The parameter $\Gamma_{k l}=\Gamma_{l k}=1 / T_{2}(k l)$ is the phase relaxation rate constant of the level pair $(k l), \Gamma_{k k}=1 / T_{1}(k)$ is the total decay rate constant of the level $k$, and $\gamma_{m k}$ is the rate constant of incoherent population transfer (feeding) from level $m$ to level $k$. The conservation of total population $(\operatorname{Tr}\{\rho\}=1)$ requires that the feeding parameters obey the equation

$$
\sum_{k} \gamma_{m k}=\Gamma_{m m} \text {. }
$$

Eq. (20) is a system of coupled differential equations for the matrix elements of $\rho(t)$. The coupling due to the interaction $V(t)$ can be removed by a perturbative treatment. For this purpose $\rho(t)$ is expanded in a Taylor series in powers of the perturbation,

$\rho(t)=\rho^{(0)}(t)+\rho^{(1)}(t)+\rho^{(2)}(t)+\rho^{(3)}(t)+\ldots$.

Then the Liouville equation for a particular order $\rho^{(n)}(t)$ is

$\dot{\rho}(n)=-\Gamma_{k l} \rho(n)+f(t)$

$f(t)=\frac{\mathrm{i}}{\hbar}\left[\rho^{(n-1)}, V\right]_{k l}+\delta_{k l} \sum_{m \neq k} \gamma_{m k} \rho_{m m}^{(n)}$.

The differential equations of the diagonal elements are still coupled through the feeding parameters. In case of an off-diagonal element $f(t)$ contains only matrix elements of $\rho$ in a lower order and does not depend on the matrix element under consideration. Hence it is simply a function of time, and eq. (25) can be integrated to yield

$\rho l^{(n)}(t)=\exp \left(-\Gamma_{k l} t\right) \int_{-\infty}^{t} \mathrm{~d} t^{\prime} \exp \left(\Gamma_{k l} t^{\prime}\right) f\left(t^{\prime}\right)$.

We note that eqs. (25) and (27) are linear in $f(t)$. If $f(t)$ is the sum of several terms, the integral in eq. (27) can be written as the corresponding sum. In this way the off-diagonal elements of $\rho^{(n)}(t)$ can be calculated iteratively beginning with a suitable zero-order solution, usually $\rho_{a a}^{(0)}=1$, where the index $a$ labels the ground state. These iterations can be continued to any desired order as long as no step involves a diagonal element of $\rho$. This is possible for the lowest order terms describing sum- and difference-frcquency mixing as well as CARS and CSRS. However, populations do occur in the calculation of grating experiments, polarization spectroscopy, transient hole-burning, and higher order contributions to CARS and CSRS. In order to apply the formalism to all matrix elements the feeding parameters are usually neglected in the diagrammatic perturbation theories. This certainly introduces an error and a correct description is desirable.

For the two-level system feeding can be treated exactly [13]. The diagonal matrix elements of the relaxation operator are

$(\dot{\rho})_{a a}^{\mathbf{R}}=-\Gamma_{a a} \rho_{a a}+\gamma_{b a} \rho_{b b}, \quad(\dot{\rho})_{b b}^{\mathrm{R}}=-\Gamma_{b b} \rho_{b b}+\gamma_{a b} \rho_{a a}$. 
Since the total population is conserved, we have $\rho_{a a}^{(n)}=-\rho_{b b}^{(n)}$ in all orders $n \geqslant 1$. Each level has only the other to decay to, so that $\gamma_{a b}=\Gamma_{a a}$ and $\gamma_{b a}=\Gamma_{b b}$. Consequently, eq. (28) can be rewritten in the form

$\left(\dot{\rho}^{(n)}\right)_{a a}^{\mathbf{R}}=-\tilde{\Gamma}_{a a} \rho_{a a}^{(n)}, \quad\left(\dot{\rho}^{(n)}\right) \underset{b b}{\mathrm{R}}=-\tilde{\Gamma}_{b b} \rho b_{b}^{(n)}$,

with $\tilde{\Gamma}_{a a}=\tilde{\Gamma}_{b b}=\Gamma_{a a}+\Gamma_{b b}$. Hence the two differential equations are decoupled by formally neglecting feeding and replacing the decay rate constants with the effective rate constant $\tilde{\Gamma}_{a a}$.

For multilevel systems such a transformation to a decoupled set of differential equations is in general not possible. However, the technique presented in eq. (27) can still be used to calculate the diagonal matrix elements one at a time if a hierarchical level ordering can be found. This is true if the levels can be arranged in an order $a<b<c \ldots$ such that $\gamma_{m k}=0$ for $m<k$. This means that feeding occurs only from higher levels to lower ones, and such a situation is not unlikely to occur at low temperatures.

For a hierarchically ordered system the differential equation of the highest level populated, say $\rho_{z z}^{(n)}$, contains no feeding contribution and can hence be solved in the manner of eq. (27). In the differential equation for the population of the next lower level $\rho_{y y}^{(n)}$ in the same order of perturbation theory, the term $\gamma_{z y} \rho_{z z}^{(n)}$ is now an already known function of time. Hence the differential equation is again of the form of eq. (25) and can be integrated. In this way all diagonal elements can be calculated from top to bottom. A strategy for the perturbative calculation of the susceptibility or the response function of a hierarchically ordered molecular system involves the steps shown in fig. 1. This technique can be incorporated into the diagrammatic method of perturbation theory, thereby allowing for the specific calculation of only those terms which are of interest for a particular experimental situation.

\section{Generation of diagrams}

A straightforward application of the strategy outlined in fig. 1 will yield all matrix elements of $\rho^{(n)}$, each containing all Fourier components that can be generated by an $n$-fold combination of the Fourier components contained in the interaction operator $V$. A particular experiment, howcver, is often described by only a single Fourier component of a single matrix element $\rho\left(k_{l}^{(n)}\right.$. A strategy that allows us to specifically calculate only the terms of interest is provided by the technique of diagrammatic perturbation theory. To understand the basic principle behind it, let us write the operators $\rho$ and $V$ as sums of elementary operators,

$\rho^{(n)}=\sum_{k l} \hat{\rho}_{k l}^{(n)}$

$\hat{\rho}_{k l}^{(n)}=|k>\rho(n)<l|$,

$V=\sum_{r s \alpha}\left[\hat{V}_{r s}\left(\omega_{\alpha}\right)+\hat{V}_{r s}\left(-\omega_{\alpha}\right)\right]$,

$\hat{V}_{r s}\left(\omega_{\alpha}\right)=|r\rangle V_{r s}\left(\omega_{\alpha}\right)\langle s|$.

The matrix element $V_{r s}\left(\omega_{\alpha}\right)$ in the interaction representation has the form

$V_{r s}\left(\omega_{\alpha}\right)=-\frac{1}{2} \mu_{r s} \cdot \mathscr{E}_{\alpha} \exp \left[\mathrm{i}\left(\omega_{r s}+\omega_{\alpha}\right) t\right] \Delta\left(t-\tau_{\alpha}\right)$,

where $\omega_{r s}=\left(\epsilon_{r}-\epsilon_{s}\right) / \hbar$ and $\epsilon_{r}$ is the energy of the molecular level $r$. For the calculation of susceptibilities $\Delta(t)=1$, for response functions $\Delta(t)=\delta(t)$. The commutator of a single $\left.\hat{\rho} \hat{k}^{n}\right)$ with a single $\hat{V}_{r s}\left(\omega_{\alpha}\right)$ yields two terms,

$\left[\hat{\rho}_{k l}^{(n)}, \hat{V}_{r s}\left(\omega_{\alpha}\right)\right]=|k\rangle \rho k_{l}^{(n)}\langle l|| r\rangle V_{r s}\left(\omega_{\alpha}\right)\langle s|-| r\rangle V_{r s}\left(\omega_{\alpha}\right)\langle s|| k\rangle \rho k_{l}^{(n)}\langle l|$ 


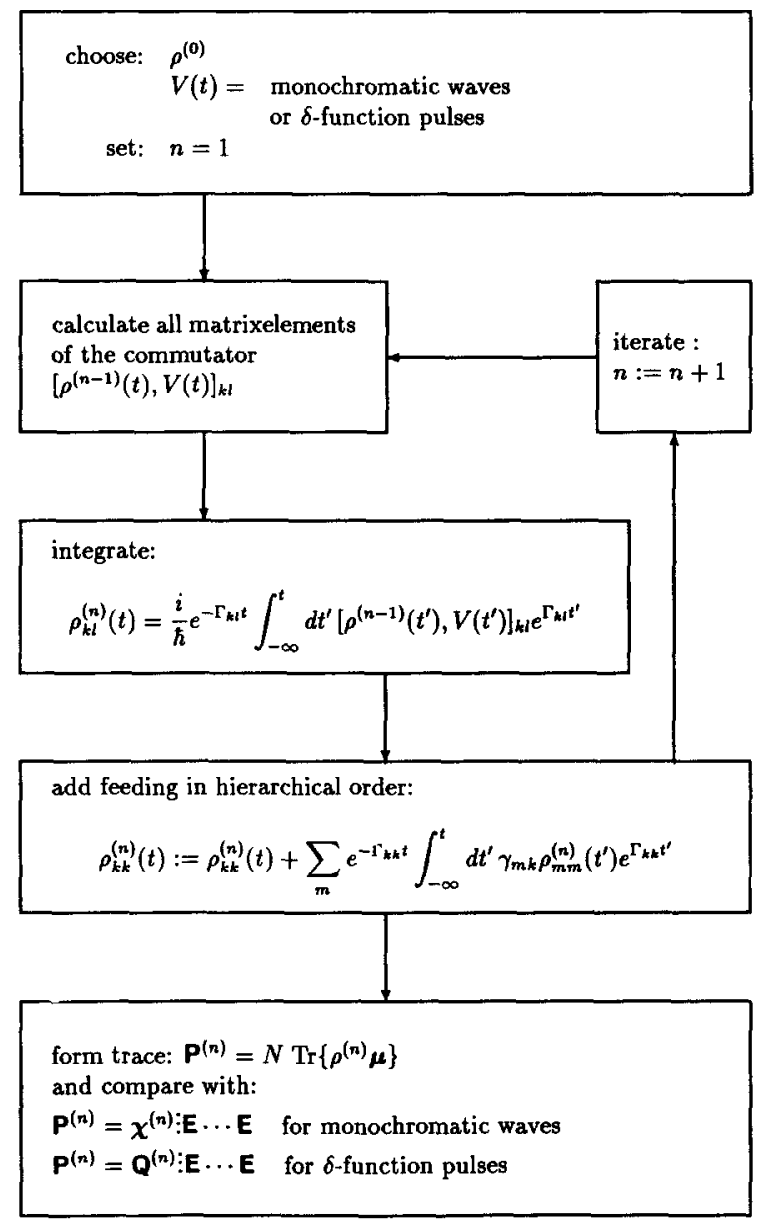

Fig. 1. Flow diagram for the perturbative calculation of the statistical operator of a multilevel system under the influence of several light ficlds. Fceding within a hicrarchically ordercd level system is considered.

If $l=r$, the bra of the first operator and the ket of the second fit together like the pieces of a domino game. Then the first term will yield a contribution to $\hat{\rho}_{k s}^{(n+1)}$, i.e. the bra-index of $\rho$ has evolved from $l$ to $s$. Similarly the second term yields a contribution to $\hat{\rho}_{r l}^{(n+1)}$ if $s=k$ and evolves the ket-index of $\rho$ from $k$ to $r$.

It should be reminded that the commutator of $\hat{\rho}_{k l}^{(n)}$ with the full operator $\hat{V}$ is the sum of eq. (35) over the indices $r, s, \alpha$ and the sign of $\omega_{\alpha}$. Hence even if a particular choice of the Fourier component has been made, the bra and ket of $\hat{\rho}_{k l}^{(n)}$ are evolved to all possible values, i.e. the result must be summed over these indices. However, in resonant situations the contribution of a single level is often dominant.

A particular sequence of propagation steps with elementary propagation operators can be graphically represented by a diagram. Each diagram leads to an additive contribution to the susceptibility or the response function. Several diagrams may contribute to the same Fourier component of the matrix element of interest. Our aim is to find all possible valid diagrams for a particular experimental situation. In a second step the susceptibility or response function is directly constructed from these diagrams.

We prefer a notation in which the framework of the diagram is the molecular level picture. The indices for the bra and ket can be marked with a full and an empty circle, respectively. Thus diagram (i) in fig. 2 corresponds to $\rho_{a a}^{(0)}$, diagram (ii) to $\rho_{b b}^{(0)}$. Since $\rho_{a a}^{(0)}$ is by far the most common initial condition, the circles are usually omitted in this case. A propagation step is represented by a vertical arrow, and the sequence of steps proceeds from left to right. An interaction operator $V_{r s}(\omega)$ is represented by an arrow pointing from level $r$ to level $s$. It 
(i)

$\mathrm{b}$

$a$

(iii)

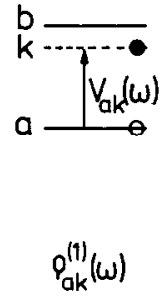

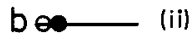
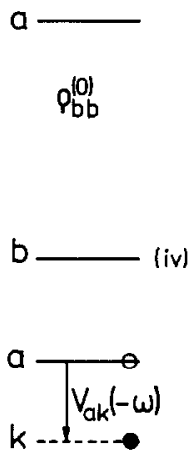

$\mathrm{p}_{\mathrm{ak}}^{(1)}(-\omega)$ (v)

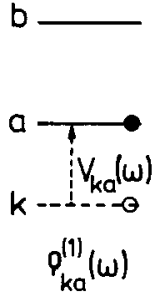

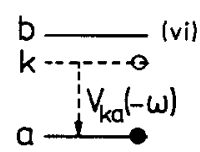

$q_{k a}^{(1)}(-w)$

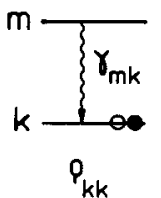

Fig. 2. Building blocks for diagrams describing perturbative propagation for a matrix element of the statistical operator. (i,ii) Initial conditions, (iii,iv) bra-propagation, (v,vi) ket-propagation, (vii) feeding.

points up if the frequency $\omega$ is positive and down when it is negative. If this operator propagates the bra side of $\rho$ the arrow is drawn full with its tail starting at the old bra-index and pointing to the new bra-index. For the propagation of the ket-index the arrow is broken with its head at the old index.

Thus a monochromatic interaction starting from $\rho_{a a}^{(0)}$ yields the four first-order diagrams (iii-vi) in fig. 2. The level $k$ is drawn as a dotted line in order to indicate that the final results still must be summed over this index. Those diagrams in which $k$ comes close to a real level $b$ give rise to resonant contributions. No real level can be below the ground state, and hence the diagrams (iv) and (v) are neglected within the rotating wave approximation (RWA). Since the indices of the interaction operator are obvious from the beginning and end of the arrow, and the sign of the frequency is known from its direction, it is sufficient to label each arrow only by the index of the frequency involved.

Successive arrows must begin where the previous of the same type left off. The total number of full and broken arrows in the diagram is equal to the perturbative order of the matrix element represented by this diagram, and the sum of the corresponding interaction frequencies indicate its Fourier component. The actual bra and ket indices of a certain diagram are given by the head of the latest full arrow and the tail of the latest broken arrow. If both indices are equal, the diagram represents a population term. In this case a propagation via feeding is possible, where $\gamma_{m k}$ is represented by a curled arrow from $m$ to $k$, as shown in the last diagram of fig. 2. This interaction propagates both indices from $m$ to $k$. The result is again a population term of the same perturbative order.

As an example we discuss a three-colour grating experiment within a three-level system. Two lasers with frequencies $\omega_{1}$ and $\omega_{2}$ are resonant with the transition from the ground state $a$ to the upper state $c$, and the third laser is resonant with the transition from $a$ to the intermcdiatc levcl $b$. Wc arc interested in the third-order response of the system at the frequency $\omega_{4}=\omega_{1}-\omega_{2}+\omega_{3}$. Within the rotating wave approximation only the three interaction matrix elements $V_{1}=V_{a c}\left(\omega_{1}\right), V_{2}=V_{c a}\left(-\omega_{2}\right)$, and $V_{3}=V_{a b}\left(\omega_{3}\right)$ need to be considered, and each may occur only once in each diagram. Fig. 3 shows the path diagram indicating all the possible distinct 

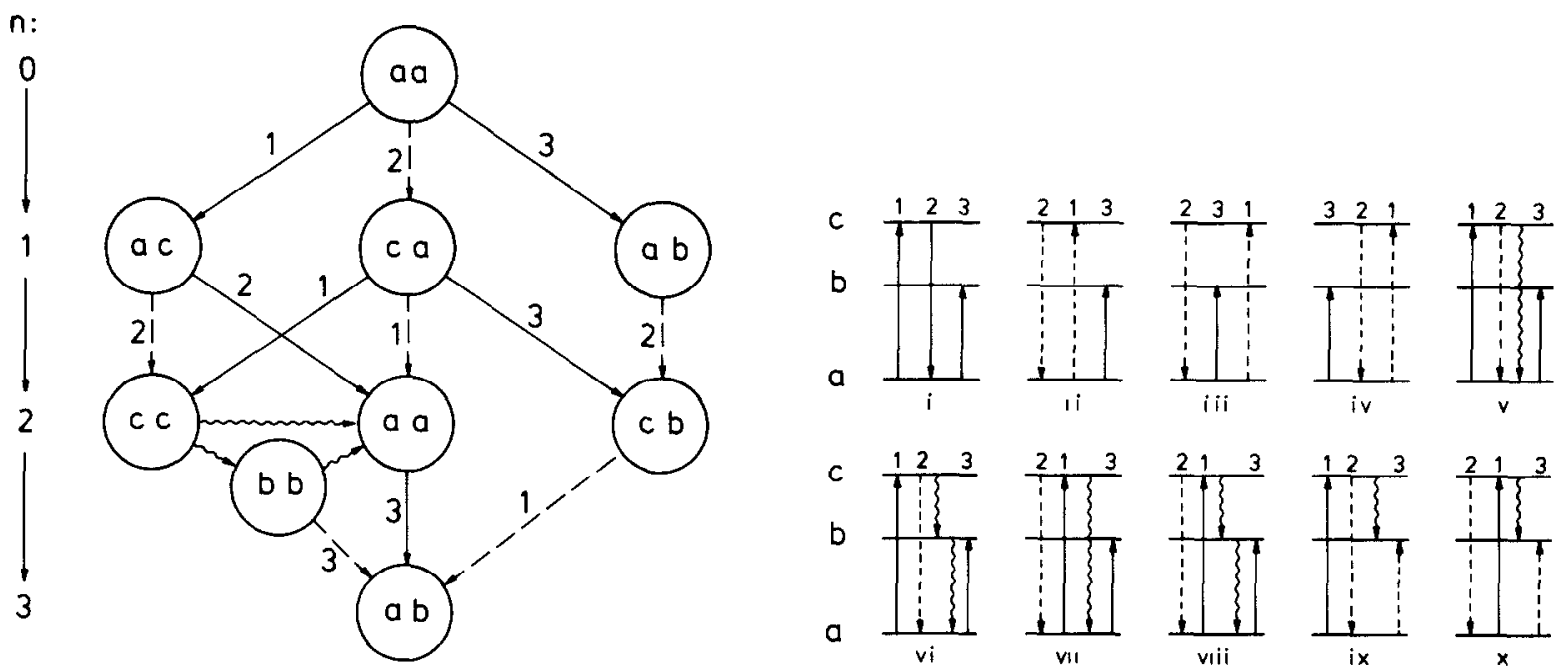

Fig. 3. Left: schematic representation of all paths in perturbation theory arising in a fully resonant three-colour grating experiment in a three-level system. Two fields with frequencies $\omega_{1}$ and $\omega_{2}$ are resonant with the $a \rightarrow c$ transition, the third with frequency $\omega_{3}$ is resonant with the $a \rightarrow b$ transition. The signal of interest is the response at frequency $\omega_{1}-\omega_{2}+\omega_{3}$. Right: the corresponding diagrams.

propagation sequences and below the resulting diagrams. All paths start from the zero-order density matrix with the only nonvanishing element $\rho_{a a}^{(0)}=1$. In the first step $V_{1}$ and $V_{3}$ can only be applied to the bra side, and $V_{2}$ to the ket side, leading to three first order terms $\rho_{a c}^{(1)}\left(\omega_{1}\right), \rho_{c a}^{(1)}\left(-\omega_{2}\right)$, and $\rho_{a b}^{(1)}\left(\omega_{3}\right)$. Propagation of $\rho_{a c}^{(1)}\left(\omega_{1}\right)$ on either the bra or the ket side with $V_{2}$ yields contributions to the populations $\rho_{a a}^{(2)}\left(\omega_{1}-\omega_{2}\right)$ and $\rho_{c c}^{(2)}\left(\omega_{1}-\omega_{2}\right)$. Other contributions to these populations result from the application of $V_{1}$ to both sides of $\rho_{c a}^{(1)}\left(-\omega_{2}\right)$. A coherence $\rho_{c b}^{(2)}\left(\omega_{3}-\omega_{2}\right)$ also occurs through ket-propagation of $\rho_{c a}^{(1)}\left(-\omega_{2}\right)$ with $V_{3}$ and bra-propagation of $\rho_{a b}^{(1)}\left(\omega_{3}\right)$ with $V_{2}$. In third order no propagation of $\rho_{c c}^{(2)}\left(\omega_{1}-\omega_{2}\right)$ is possible with the available coupling terms. The two paths leading to $\rho_{a a}^{(2)}\left(\omega_{1}-\omega_{2}\right)$ both used already the couplings $V_{1}$ and $V_{2}$, so only $V_{3}$ can be applied to yield a contribution to $\rho_{a b}^{(3)}\left(\omega_{1}-\omega_{2}+\omega_{3}\right)$. This yields the two diagrams (i) and (ii). Similarly two contributions to the same matrix element arise from the ket-propagation of $\rho_{c h}^{(2)}\left(\omega_{3}-\omega_{2}\right)$ with $V_{1}$, shown as diagrams (iii) and (iv). With the neglect of feeding no more diagrams are possible. Feeding connects $\rho_{c c}^{(2)}$ directly with $\rho_{a a}^{(2)}$ and also indirectly via $\rho(2)$. Since two paths lead to $\rho_{c c}^{(2)}$, feeding yields four additional paths to $\rho_{a a}^{(2)}$ which consequently yield four diagrams ( $v$-viii). The occurrence of $\rho_{b b}^{(2)}$ finally allows for a new ket-propagation to $\rho_{a b}^{(3)}\left(\omega_{1}-\omega_{2}+\omega_{3}\right)$ via $V_{3}$, giving the diagrams (ix) and (x) in fig. 3. This example shows that even in a seemingly simple situation the number of diagrams can be large, and increases even more when feeding is considered. It is therefore important to find a strategy which guarantees that all necessary diagrams have been found. As will be shown later, such a strategy can be designed and programmed.

\section{Calculation of susceptibilities}

The contributions to the susceptibility are calculated from the steady-state response of the system under the influence of monochromatic waves. For a particular Fourier component of a density matrix element $\rho \mathrm{km}^{n-1)}(t)$ with oscillation frequency $\Omega$ we can make the ansatz in the interaction picture

$\rho k_{m}^{(n-1)}(t)=r_{k m}^{n-1)} \exp \left[\mathrm{i}\left(\omega_{k m}+\Omega\right) t\right]$. 
Propagation of the bra-index with an interaction $V_{m l}\left(\omega_{\alpha}\right)$ in the form given in eq. (34) yields a contribution to $\rho_{k l}^{(n)}(t)$. It is found through integration of eq. (27) with

$f(t)=-\frac{\mathrm{i}}{2 \hbar} r_{k m}^{(n-1)} \boldsymbol{\mu}_{m l} \cdot \mathscr{E}_{\alpha} \exp \left[\mathrm{i}\left(\omega_{k l}+\Omega+\omega_{\alpha}\right) t\right]$,

where we have used $\omega_{k m}+\omega_{m l}=\omega_{k l}$. The result is

$\rho \rho_{k l}^{(n)}(t)=r_{k m}^{(n-1)} \frac{-\mu_{m l}}{\hbar\left(\omega_{k l}+\Omega+\omega_{\alpha}-\mathrm{i} \Gamma_{k l}\right)} \cdot \frac{\mathscr{E}_{\alpha}}{2} \exp \left[\mathrm{i}\left(\omega_{k l}+\Omega+\omega_{\alpha}\right) t\right]$.

This has again the form of eq. (36) with the new oscillation frequency being $\Omega^{\prime}=\Omega+\omega_{\alpha}$ and the new amplitude

$r k_{k l}^{n)}=r_{k m}^{(n-1)} \frac{-\mu_{m l}}{h\left(\omega_{k l}+\Omega+\omega_{\alpha}-\mathrm{i} \Gamma_{k l}\right)} \cdot \frac{\mathscr{E}_{\alpha}}{2}$

Thus the choice of functional form remains consistent. Usually the zero-order density matrix has the oscillation frequency $\Omega=0$. Then the oscillation frequency $\Omega^{\prime}$ of $\rho_{k l}^{(n)}(t)$ is the sum of the $n$ field frequencies of the $n$ propagation steps with the interaction operator. After the last step in the propagation sequence the susceptibility is calculated by taking the trace $\operatorname{Tr}\{N \rho \mu\}$. In the case of eq. (38) this cancels the factor $\exp \left(i \omega_{k l} t\right)$ and adds a factor $N \mu_{l k}$. We know that the result is related to the macroscopic susceptibility by

$N \rho_{k l}^{(n)}(t) \mu_{l k}=\chi^{(n)}\left(\omega_{n}, \ldots, \omega_{1}\right) \vdots \prod_{\alpha}\left[\frac{1}{2} \mathscr{E}_{\alpha} \exp \left(\mathrm{i} \omega_{\alpha} t\right)\right]$

Comparison with eq. (38) shows that the bra-propagation of $\rho_{k m}$ with $V_{m l}\left(\omega_{\alpha}\right)$ yields a multiplicative factor

$\frac{-\mu_{m l}^{(\alpha)}}{\hbar\left(\omega_{k l}+\sum \omega\right.}-\overline{\left.\mathrm{i} \Gamma_{k l}\right)}$

to the susceptibility. The symbol $\otimes$ indicates a tensorial multiplication, and $\sum \omega$ is the sum of all field frequencies occurring in the diagram up to and including the step considered. The superscript in $\mu_{m l}^{(\alpha)}$ identifies the field component whose polarization vector forms the scalar product with it. When the susceptibility is expressed in Cartesian coordinates, this index labels the Cartesian index of $\chi$ associated with the field with frequency $\omega_{\alpha}$. If we perform the same calculation for a ket-propagation, e.g. of $\rho_{m l}$ with $V_{k m}\left(\omega_{\alpha}\right)$ leading to $\rho_{k l}$, the contribution to the susceptibility is

$\frac{\mu_{k m}^{\alpha} \otimes}{\hbar\left(\omega_{k l}+\sum \omega-\mathrm{i} \Gamma_{k l}\right)}$

Except for the change in sign we obtain the same result: the index pair occurring in the denominator is that of the density matrix element generated in this propagation step, and the index pair occurring in the numerator is that of the interaction.

Finally we consider the propagation from $\rho_{m m}^{(n)}$ by a feeding step with rate constant $\gamma_{m k}$ to $\rho_{k k}^{(n)}$. Taking again an ansatz according to eq. (36) for $\rho_{m m}^{(n)}$ we have to integrate eq. (27) with the function

$f(t)=\gamma_{m k} r_{m m}^{(n)} \exp \left(\mathrm{i} \sum \omega t\right)$

The result is that this propagation also yields a multiplicative factor to the susceptibility, namely

$\frac{-\mathrm{i} \gamma_{m k}}{\sum \omega-\mathrm{i} \Gamma_{k k}}$

Again the index pair in the numerator is that of the coupling and the index pair in the denominator is that of the 
generated matrix element. The graphical representation of the three types of propagation steps in the diagrams and their multiplicative contributions to the susceptibility are summarized in fig. 4 .

The contribution to the susceptibility represented by a diagram can therefore be obtained in the following way:

- The first factor is the starting zero-order matrix element of the density matrix, usually $\rho_{a a}^{(0)}=1$.

- Each propagation step yields a factor according to the prescription given above.

- The last factor $N \mu_{l k}^{(\alpha)}$ results from taking the trace $\operatorname{Tr}\{N \rho \mu\}$. The index pair $(l k)$ is that of the final density matrix element in reversed order, and $\alpha$ labels the first Cartesian index of $\chi$ which refers to the Cartesian components of the non-linear optical polarization, i.e. it gives the polarization direction of the created light field.

- The resulting expression must be summed over the indices of all levels occurring in the diagram. If resonances occur, the sum can be truncated to the largest contribution.

As examples we give below the susceptibilities for the two third-order diagrams in fig. 5 describing CARS and CSRS with a ground state vibrational resonance within the rotating wave approximation,

$\chi_{i j k l}^{\mathrm{CARS}}\left(\omega_{1},-\omega_{2}, \omega_{1}\right)=\frac{N}{\hbar^{3}} \sum_{c d} \rho_{a a}^{(0)} \frac{-\mu_{a c}^{(l)} \otimes \mu_{c b}^{(k)} \otimes \mu_{b d}^{()} \otimes \mu_{d a}^{(i)}}{\left(\omega_{a c}+\omega_{1}-\mathrm{i} \Gamma_{a c}\right)\left(\omega_{a b}+\omega_{1}-\omega_{2}-\mathrm{i} \Gamma_{a b}\right)\left(\omega_{a d}+2 \omega_{1}-\omega_{2}-\mathrm{i} \Gamma_{a d}\right)}$,

$\chi_{i j k l}^{\mathrm{CSRS}}\left(\omega_{1}, \omega_{1},-\omega_{2}\right)=\frac{N}{\hbar^{3}} \sum_{c d} \rho_{a a}^{(0)} \frac{-\mu_{d a}^{(l)} \otimes \mu_{b d}^{(k)} \otimes \mu_{a c}^{()} \otimes \mu_{c b}^{(b)}}{\left(\omega_{d a}-\omega_{2}-\mathrm{i} \Gamma_{d a}\right)\left(\omega_{b a}+\omega_{1}-\omega_{2}-\mathrm{i} \Gamma_{b a}\right)\left(\omega_{b c}+2 \omega_{1}-\omega_{2}-\mathrm{i} \Gamma_{b c}\right)}$.

The sum over the levels $c$ and $d$ can be truncated to those levels fulfilling the resonance conditions expressed in

\begin{tabular}{|c|c|c|}
\hline Diagram & Susceptibility & Response function \\
\hline 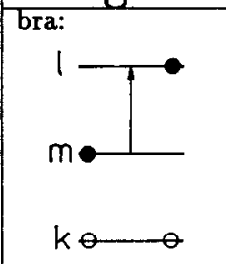 & $\frac{-\mu_{m l}^{(\alpha)} \otimes}{\hbar\left(\omega_{k l}+\sum \omega-i \Gamma_{k l}\right)}$ & $\begin{array}{c}\frac{-i}{\hbar}\left(\mu_{m l}^{(\alpha)}\left(t_{n}\right) \otimes\right) \\
\exp \left\{-\left(i \omega_{k l}+\Gamma_{k l}\right)\left(t_{n+1}-t_{n}\right)\right\}\end{array}$ \\
\hline 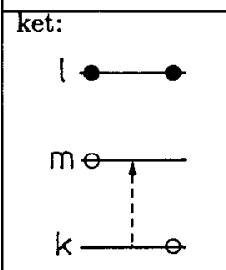 & $\frac{\boldsymbol{\mu}_{k m}^{(\alpha)} \otimes}{\hbar\left(\omega_{k l}+\sum \omega-i \Gamma_{k l}\right)}$ & $\begin{array}{c}\frac{i}{\hbar}\left(\boldsymbol{\mu}_{k m}^{(\alpha)}\left(t_{n}\right) \otimes\right) \\
\exp \left\{-\left(i \omega_{k l}+\Gamma_{k l}\right)\left(t_{n+1}-t_{n}\right)\right\}\end{array}$ \\
\hline feeding: & $\frac{-i \gamma_{m k}}{\sum \omega-\imath \Gamma_{k k}}$ & $\begin{array}{c}\exp \left\{-\Gamma_{x x}\left(t_{n+1}-t_{n}\right)\right\} \rightarrow \\
\frac{\gamma_{m k}}{\Gamma_{x x}-\Gamma_{k k}} \\
{\left[\exp \left\{-\Gamma_{k k}\left(t_{n+1}-t_{n}\right)\right\}\right.} \\
\left.-\exp \left\{-\Gamma_{x x}\left(t_{n+1}-t_{n}\right)\right\}\right]\end{array}$ \\
\hline
\end{tabular}

Fig. 4. Summary of the three types of propagators in diagrams and their contributions to susceptibilities and response functions. 
(i)

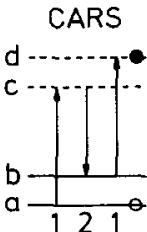

(iii)

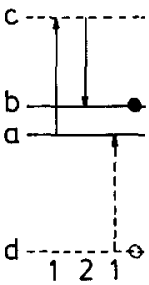

CSRS
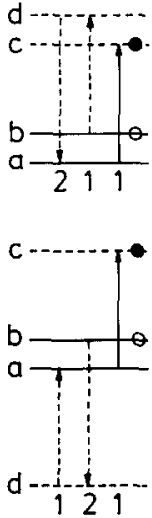

(ii)

(iv) (v)

CARS

CSRS

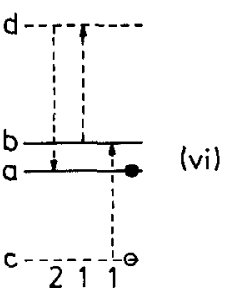

(vii)
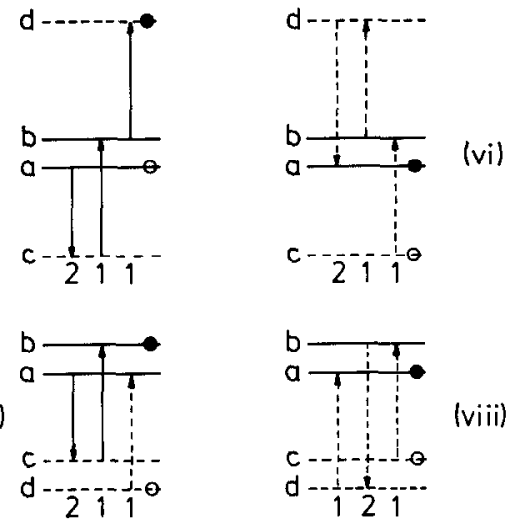

Fig. 5. Third-order diagrams displaying the resonance between the ground state $a$ and a vibrational state $b$ in CARS and CSRS. The diagrams (i) and (ii) remain when the rotating wave approximation is applied.

the first and third resonance denominators. The formula generated from the diagram, without summing over indices, represents the fully resonant case. If only the vibrational $\omega_{a b}$-resonance occurs, the double sum must be performed. The result can be rewritten as

$\chi_{i j k l}^{\mathrm{CARS}}\left(\omega_{1},-\omega_{2}, \omega_{1}\right)=\frac{-N \rho_{a a}^{(0)} \beta_{i j}^{* \mathrm{CARS}} \alpha_{k l}^{\mathrm{CARS}}}{\hbar\left(\omega_{a b}+\omega_{1}-\omega_{2}-\mathrm{i} \Gamma_{a b}\right)}$,

$\chi_{i j k l}^{\mathrm{CSRS}}\left(\omega_{1},-\omega_{2}, \omega_{1}\right)=\frac{-N \rho_{a a}^{(0)} \beta_{b j}^{\text {CSRs }} \alpha_{k l}^{* \text { cSRs }}}{\hbar\left(\omega_{a b}+\omega_{1}-\omega_{2}+\mathrm{i} \Gamma_{a b}\right)}$.

Here the polarizabilities $\alpha$ and $\beta$ are defined as

$\beta_{i j}^{\text {CARS }}=\sum_{c} \frac{\mu_{a c}^{(i)} \mu_{c b}^{(b)}}{\hbar\left(\omega_{a c}+\omega_{4}+\mathrm{i} \Gamma_{a c}\right)}$,

$\beta_{y}^{\mathrm{CSRS}}=\sum_{c} \frac{\mu_{a c}^{()} \mu_{c b}^{(l)}}{\hbar\left(\omega_{b c}+\omega_{4}-\mathrm{i} \Gamma_{b c}\right)}$,

$\alpha_{i j}^{\mathrm{CARS}}=\sum_{c} \frac{\mu_{a c}^{()} \mu_{c b}^{(t)}}{\hbar\left(\omega_{a c}+\omega_{1}-\mathrm{i} \Gamma_{a c}\right)}$

$\alpha_{i j}^{\text {CSRS }}=\sum_{c} \frac{\mu_{a c}^{(i)} \mu_{c b}^{()}}{\hbar\left(\omega_{a c}+\omega_{2}-\mathrm{i} \Gamma_{a c}\right)}$,

with $\omega_{4}=2 \omega_{1}-\omega_{2}$. When the transition dipoles are real and all field frequencies are far from electronic resonances, $\boldsymbol{\beta}^{+ \text {CARS }}=\alpha^{\text {CSRS }}$, and near the vibrational resonance $\beta^{\text {CSRS }}=\alpha^{\text {CARS }}$. In this case the dephasing parameters may be neglected and all polarizabilities become real. Under these conditions the validity of the rotating wave approximation must be checked, and indeed three further diagrams can be found each for CARS and for CSRS that display the vibrational resonance and yicld contributions to the polarizabilities. These are shown as diagrams iii to viii in fig. 5 . When all four contributions to each process are added the formula for the susceptibility has again the form of eqs. (47) and (48), but with the polarizabilities given by

$\beta_{i j}^{\mathrm{CARS}}=\sum_{c}\left(\frac{\mu_{a c}^{(i)} \mu_{c b}^{()}}{\hbar\left(\omega_{a c}+\omega_{4}+\mathrm{i} \Gamma_{a c}\right)}+\frac{\mu_{a c}^{()} \mu_{c b}^{(l)}}{\hbar\left(\omega_{b c}-\omega_{4}-\mathrm{i} \Gamma_{b c}\right)}\right)$, 
$\beta_{i j}^{\mathrm{CSRS}}=\sum_{c}\left(\frac{\mu_{a c}^{(j)} \mu_{c b}^{(j)}}{\hbar\left(\omega_{b c}+\omega_{4}-\mathrm{i} \Gamma_{b c}\right)}+\frac{\mu_{a c}^{(i)} \mu_{c b}^{(j)}}{\hbar\left(\omega_{a c}-\omega_{4}+\mathrm{i} \Gamma_{a c}\right)}\right)$

$\alpha_{i j}^{\mathrm{CARS}}=\sum_{c}\left(\frac{\mu_{a c}^{()} \mu_{c b}^{(l)}}{\hbar\left(\omega_{a c}+\omega_{1}-\mathrm{i} \Gamma_{a c}\right)}+\frac{\mu_{a c}^{(l)} \mu_{c b}^{(b)}}{\hbar\left(\omega_{a c}-\omega_{2}-\mathrm{i} \Gamma_{a c}\right)}\right)$

$\alpha_{i j}^{\mathrm{CSRS}}=\sum_{c}\left(\frac{\mu_{a c}^{(i)} \mu_{c b}^{())}}{\hbar\left(\omega_{a c}+\omega_{2}-\mathrm{i} \Gamma_{a c}\right)}+\frac{\mu_{a c}^{(j)} \mu_{c b}^{(l)}}{\hbar\left(\omega_{a c}-\omega_{1}-\mathrm{i} \Gamma_{a c}\right)}\right)$

These equations were already used in a previous paper [30] without derivation.

Strictly speaking only dc-Fourier components of the equilibrium populations are allowed as starting values of the zero-order density matrix, since the susceptibility refers to the equilibrium system. Hence, for a hierarchically ordered multi-level system only $\rho_{a a}^{(0)}=1$ is valid. The only exception is a system in which populations are restricted to two levels and which can be treated according to eq. (29). However, it may happen that the lifetime $T_{1}(k)=1 / \Gamma_{k k}$ of an excited state $k$ is much longer than all other decay parameters involved in the propagation paths for a particular diagram starting from that level. In this case an initial condition $\rho{ }_{k k}^{(0)} \neq 0$ is meaningful for the description of experiments on a timescale shorter than $T_{1}(k)$.

It should be noted that the contribution of an individual diagram can diverge if it involves a matrix clement of the ground state in the evolution sequence. This yields a denominator $\sum \omega-\mathrm{i} \Gamma_{a a}$, which vanishes if a dc component is considered and infinite lifetime is assumed. This must of course be an artifact, and it turns out that the divergence cancels with other terms generated by the same time ordering of perturbations, but including feeding.

\section{Calculation of response functions}

For the calculation of the response function represented by a diagram we have to replace the temporal envelope in the interaction operator of eq. (34) with a $\delta$-function. Then we must consider the effect that each of the three possible propagation steps has on a particular density matrix element, e.g. $\rho_{\mathrm{km}}^{(n-1)}(t)$. This density matrix element is the result of $(n-1)$ impulsive interactions, the last occurring at time $t_{n-1}$. It is obvious from the Liouville equation that after this time, i.e. in the absence of an interaction, the density matrix element will decay exponentially with rate constant $\Gamma_{k m}$. Hence a reasonable ansatz which, as we will see, remains self-consistent through all evolution steps is

$$
\begin{aligned}
\rho_{k m}^{(n-1)}(t) & =r_{k m}^{(n-1)} \exp \left[-\Gamma_{k m}\left(t-t_{n-1}\right)\right], & & \text { if } t>t_{n-1}, \\
& =0, & & \text { otherwise, }
\end{aligned}
$$

with an amplitude $r_{\mathrm{km}}^{(n-1)}$ constant in time. We consider again propagation of the bra-index with an interaction $V_{m l}\left(\omega_{\alpha}\right)$ which is now a $\delta$-function impulse centered at time $t_{n}$,

$V_{m l}\left(\omega_{\alpha}\right)=-\frac{1}{2} \mu_{m l}(t) \cdot \mathscr{E}_{\alpha} \exp \left[\mathrm{i}\left(\omega_{m l}+\omega_{\alpha}\right) t\right] \delta\left(t-t_{n}\right)$.

Here the time dependence of the dipole operator accounts for a possible change in orientation of the molecule with time. The integral of eq. (27) yields then for $\left.\rho k_{l}^{n}\right)(t)$ an expression of the same form as eq. (57),

$$
\begin{aligned}
& \rho k_{l}^{(n)}(t)=r_{k m}^{(n-1)}\left(\frac{-\mathrm{i}}{2 \hbar} \mu_{m l}\left(t_{n}\right) \cdot \mathscr{E}_{\alpha}\right) \exp \left[-\Gamma_{k m}\left(t_{n}-t_{n-1}\right)\right] \exp \left[-\Gamma_{k l}\left(t-t_{n}\right)\right] \exp \left[\mathrm{i}\left(\omega_{m l}+\omega_{\alpha}\right) t_{n}\right], \\
& \text { if } t>t_{n-1} \text {. }
\end{aligned}
$$

We know that after taking the trace $\operatorname{Tr}\{N \rho \mu\}$ the final expression must be of the form 
$N \rho\left(_{k l}^{(n)}(t) \mu_{l k}=\mathbf{Q}^{(n)}\left(t ; t_{n}, \ldots, t_{1}\right) \vdots \prod_{\alpha=1}^{n}\left(\frac{1}{2} \mathscr{E}_{\alpha} \exp \left(\mathrm{i} \omega_{\alpha} t_{\alpha}\right)\right)\right.$

Hence the factor $\frac{1}{2} \mathscr{E}_{\alpha} \exp \left(\mathrm{i} \omega_{\alpha} t_{n}\right)$ in eq. (59) does not contribute to the generalized response function. The contribution from the bra-propagation step is therefore found if we first replace $t$ with $t_{n}$ in the parent expression and then multiply with

$\frac{-\mathrm{i}}{\hbar}\left[\mu_{m l}^{(\alpha)}\left(t_{n}\right) \otimes\right] \exp \left[-\Gamma_{k l}\left(t-t_{n}\right)+\mathrm{i} \omega_{m l} t_{n}\right]$,

where the symbol $\otimes$ again indicates tensorial multiplication with the term from the next propagation step. At the end of the propagation steps the trace $\operatorname{Tr}\{N \rho \mu\}$ will yield a final factor $N \mu_{k k}^{(\alpha)}(t) \exp \left\{\mathrm{i} \omega_{l k} t\right\}$ : It is easily seen that the same result is found for the response function when each bra-propagation produces a factor

$\frac{-\mathrm{i}}{\hbar}\left[\mu_{m l}^{(\alpha)}\left(t_{n}\right) \otimes\right] \exp \left[-\left(\mathrm{i} \omega_{k l}+\Gamma_{k l}\right)\left(t_{n+1}-t_{n}\right)\right]$,

and only $N \mu_{k}^{(\alpha)}(t)$ is considered as the contribution of the trace. In the final result $t_{n+1}$ is interpreted as $t$, i.e. the time argument of the generated polarization. (Eq. (62) is directly obtained when the calculation is performed in the Schrödinger picture.)

For the ket-propagation we obtain the same result, except for a change of sign. E.g., the propagation of $\rho_{m l}^{(n-1)}$ with $V_{k m}\left(\omega_{\alpha}\right)$ at time $t_{n}$ leading to $\rho k_{l}^{(n)}$ yields the factor

$\frac{\mathrm{i}}{\hbar}\left[\mu_{k m}^{(\alpha)}\left(t_{n}\right) \otimes\right] \exp \left[-\left(\mathrm{i} \omega_{k l}+\Gamma_{k l}\right)\left(t_{n+1}-t_{n}\right)\right]$.

In both equations, (62) and (63), the index pair of the dipole operator is that of the interaction and the index pair in the exponential is that of the generated density matrix element.

The third type of propagation is through feeding. If we describe the population of the upper level by a single exponential decay

$\rho_{m m}^{(n)}(t)=r_{m m}^{(n)} \exp \left[-\Gamma_{m m}\left(t-t_{n}\right)\right]$

for times $t>t_{n}$, the integration of eq. (27) for $\rho k_{k}^{(n)}$ generated through feeding with rate constant $\gamma_{m k}$ is found to be

$\rho \sum_{k k}^{(n)}(t)=r_{m m}^{(n)} \frac{\gamma_{m k}}{\Gamma_{m m}-\Gamma_{k k}}\left\{\exp \left[-\Gamma_{k k}\left(t-t_{n}\right)\right]-\exp \left[-\Gamma_{m m}\left(t-t_{n}\right)\right]\right\}$

Hence this propagation step could be described by a multiplicative factor of

$\frac{\gamma_{m k}}{\Gamma_{m m}-\Gamma_{k k}}\left\{1-\exp \left[\left(\Gamma_{k k}-\Gamma_{m m}\right)\left(t-t_{n}\right)\right]\right\}$.

However, since feeding replaced the single exponential decay with a double exponential, one rising and one falling, eq. (64) is in fact not the general ansatz for a population term. The general expression for a diagram of a population term is a sum of several exponentials if it contains several feeding steps. Propagation is then not described by a single factor. The general rule is instead a substitution rule: the formula of the generated population is found by replacing in the parent formula all exponentially decaying terms with the time argument $\left(t_{n+1}-t_{n}\right)$ according to the rule

$\exp \left[-\Gamma_{x x}\left(t_{n+1}-t_{n}\right)\right] \rightarrow \frac{\gamma_{m k}}{\Gamma_{x x}-\Gamma_{k k}}\left\{\exp \left[-\Gamma_{k k}\left(t_{n+1}-t_{n}\right)\right]-\exp \left[-\Gamma_{x x}\left(t_{n+1}-t_{n}\right)\right]\right\}$ 
In the special case $\Gamma_{x x}=\Gamma_{k k}$ the substitution rule is

$\exp \left[-\Gamma_{x x}\left(t_{n+1}-t_{n}\right)\right] \rightarrow \gamma_{m k} \exp \left[-\Gamma_{k k}\left(t_{n+1}-t_{n}\right)\right]\left(t_{n+1}-t_{n}\right)$.

This concludes the derivation of propagation rules for the response functions. They are summarized together with the corresponding rules for the susceptibilities in fig. 4 .

The calculation of the contribution to the response function by a particular diagram involves the following steps:

- The first factor is the starting zero-order matrix element of the density matrix. This could be a population in a higher excited state prepared at time $t_{0}$,

$\rho_{m m}^{(0)}(t)=\rho_{m m}^{(0)}(0) \exp \left[-\Gamma_{m m}\left(t-t_{0}\right)\right]$.

- Each bra- or ket-propagation step yields a factor according to the prescription given above. A feeding step is treated according to the substitution rule.

- The last factor $N \mu_{i k}^{(\alpha)}(t)$ results from taking the trace $\operatorname{Tr}\{N \rho \mu\}$, in complete analogy to the case of susceptibilities.

- The resulting expression must in principle be summed over the indices of all levels occurring in the diagram, although this sum is often not written explicitly.

As an example and for comparison to the third-order susceptibilities for CARS and CSRS discussed in the previous section we give here the corresponding response functions:

$$
\begin{aligned}
& Q_{y k l}^{\text {CARS }}\left(t ; t_{3}, t_{2}, t_{1}\right)=\mathrm{i} \frac{N}{\hbar^{3}} \sum_{c d} \rho_{a a}^{(0)} \mu_{a c}^{(l)}\left(t_{1}\right) \otimes \mu_{c b}^{(k)}\left(t_{2}\right) \otimes \mu_{b d}^{(j)}\left(t_{3}\right) \otimes \mu_{d a}^{(l)}(t) \\
& \quad \times \exp \left[-\left(\mathrm{i} \omega_{a c}+\Gamma_{a c}\right)\left(t_{2}-t_{1}\right)-\left(\mathrm{i} \omega_{a b}+\Gamma_{a b}\right)\left(t_{3}-t_{2}\right)-\left(\mathrm{i} \omega_{a d}+\Gamma_{a d}\right)\left(t-t_{3}\right)\right], \\
& Q_{y k d}^{\mathrm{CSRS}}\left(t ; t_{3}, t_{2}, t_{1}\right)=\mathrm{i} \frac{N}{\hbar^{3}} \sum_{c d} \rho_{a a}^{(0)} \mu_{d a}^{(l)}\left(t_{1}\right) \otimes \mu_{b d}^{(k)}\left(t_{2}\right) \otimes \mu_{a c}^{(j)}\left(t_{3}\right) \otimes \mu_{c b}^{(l)}(t) \\
& \quad \times \exp \left[-\left(\mathrm{i} \omega_{d a}+\Gamma_{d a}\right)\left(t_{2}-t_{1}\right)-\left(\mathrm{i} \omega_{b a}+\Gamma_{b a}\right)\left(t_{3}-t_{2}\right)-\left(\mathrm{i} \omega_{b c}+\Gamma_{b c}\right)\left(t-t_{3}\right)\right] .
\end{aligned}
$$

Both response functions are only valid for the time sequence $t>t_{3}>t_{2}>t_{1}$. If the orientations of all dipole moments are fixed in time, the general response functions above depend only on time differences. They can therefore be written as normal response functions with the new variables $t_{t}^{\prime}=t_{t}-t$. This can be Fourier-transformed to the susceptibility, but the signs of the frequencies from the diagram must be considered. Thus

$\chi^{\mathrm{CARS}}\left(\omega_{1},-\omega_{2}, \omega_{1}\right)=\int_{-\infty}^{0} \mathrm{~d} t_{3}^{\prime} \int_{-\infty}^{t_{3}^{\prime}} \mathrm{d} t_{2}^{\prime} \int_{-\infty}^{t_{2}^{\prime}} \mathrm{d} t_{1}^{\prime} \mathbf{R}^{\mathrm{CARS}}\left(t_{3}^{\prime}, t_{2}^{\prime}, t_{1}^{\prime}\right) \exp \left[\mathrm{i}\left(\omega_{1} t_{1}^{\prime}-\omega_{2} t_{2}^{\prime}+\omega_{1} t_{3}^{\prime}\right)\right]$

yields the result of eq. (45). In the corresponding transformation for the CSRS formula the exponential factor must be $\exp \left\{\mathbf{i}\left[-\omega_{2} t_{1}^{\prime}+\omega_{1}\left(t_{2}^{\prime}+t_{3}^{\prime}\right)\right]\right\}$. In a similar way the response function can be found through the inverse transformation of the susceptibility contribution from a single diagram. Although in this case the integration ranges are not restricted as above, the result will nevertheless reflect the correct time-ordering, since the complex Lorentzian lineshape $F(\omega)$ and the single sided exponential decay $F(t)$ form a Fourier-transform pair:

$$
\begin{aligned}
F(\omega) & =\frac{-\mathrm{i}}{\omega_{0}+\omega-\mathrm{i} \Gamma}, \\
F(t) & =u(t) \exp \left[-\left(\mathrm{i} \omega_{0}+\Gamma\right) t\right], \\
u(t) & =0, \quad \text { if } t<0, \\
& =1, \quad \text { otherwise. }
\end{aligned}
$$

Therefore, each integration over frequency yields a unit step function $u(\Delta t)$. The effect of the summation over 
non-resonant levels is not directly evident from the formulas of the response functions since they do not contain the frequencies of the light fields involved. It is, however, intuitively clear that a propagation step at time $t_{n}$ leading to a non-resonant ("virtual") level must be followed immediately by the next step, and thus a term proportional to $\delta\left(t_{n+1}-t_{n}\right)$ should appear in the response function. To show this let us take eq. (47) for the CARS susceptibility in which the summation over non-resonant levels has been performed. If we transform this back into the response function with the additional assumption that the frequency dependence of the polarizabilities $\alpha$ and $\beta$ can be neglected, we obtain

$$
\begin{gathered}
\mathbf{R}^{\mathrm{CARS}}\left(t_{3}, t_{2}, t_{1}\right)=\int \frac{\mathrm{d} \omega_{1}^{\prime}}{2 \pi} \int \frac{\mathrm{d} \omega_{2}}{2 \pi} \int \frac{\mathrm{d} \omega_{1}}{2 \pi} \frac{-N \rho_{a a}^{(0)} \boldsymbol{\beta}^{* \mathrm{CARS}} \otimes \boldsymbol{\alpha}^{\mathrm{CARS}}}{\hbar\left(\omega_{a b}+\omega_{1}-\omega_{2}-\mathrm{i} \Gamma_{a b}\right)} \exp \left[-\mathrm{i}\left(\omega_{1} t_{1}-\omega_{2} t_{2}+\omega_{1}^{\prime} t_{3}\right)\right] \\
=\mathrm{i} \frac{N}{\hbar} \rho_{a a}^{(0)} \boldsymbol{\beta}^{* \mathrm{CARS}} \otimes \boldsymbol{\alpha}^{\mathrm{CARS}} \exp \left[-\left(\mathrm{i} \omega_{a b}+\Gamma_{a b}\right)\left(t_{3}-t_{2}\right)\right] u\left(t_{3}-t_{2}\right) \delta\left(t_{2}-t_{1}\right) \delta\left(t_{3}\right) .
\end{gathered}
$$

This finding can be generalized to yield the substitution rule

$$
\sum_{l} \mu_{k l}^{(\alpha)} \otimes \mu l_{m}^{(\beta)} \exp \left[-\left(\mathrm{i} \omega_{J l}+\Gamma_{J l}\right)\left(t_{\beta}-t_{\alpha}\right)\right] \rightarrow \mathrm{i} \sum_{l} \frac{\mu_{k l}^{(\alpha)} \otimes \mu_{m}^{(\beta)}}{\omega_{l l}+\Omega-\mathrm{i} \Gamma_{J l}} \delta\left(t_{\beta}-t_{\alpha}\right)
$$

where $\Omega$ is the sum of all field frequencies used in the diagram up to and including the interaction at time $t_{\alpha}$ which generates the matrix element $\rho_{j l}(\Omega)$.

If the dipoles change their orientations with time, e.g. due to rotational diffusion, the tensorial product of the dipole moments must be averaged over the orientational distribution function on the basis of an appropriate model for the rotational dynamics. For a system in thermal equilibrium the result will be a rotational correlation function which depends on time intervals only. Fourier transformation to the susceptibility is then again possible in the manner of eq. (72). In the case of exponentially decaying rotational correlation functions the corresponding rate constants will add to the dephasing rate constants in the resonance denominators which determine the linewidths of resonances. This suggests that response functions are often the more flexible choice even for the description of a frequency-domain experiment.

\section{Computer algebra implementation}

The crucial task in the calculation of the susceptibility of a multilevel system for a particular set of interactions is the complete generation of all relevant diagrams. For this purpose propagation of all initial density matrix elements with all possible distinct permutations of the interactions must be considered. In each step the possibility for a bra-evolution and a ket-evolution must be checked. Each time a diagonal element occurs in the perturbation sequence new diagrams arise from the decay (feeding) of this population to all lower levels, which can again occur directly or indirectly. Since this can lead to an enormous number of possible combinations, of which only a small fraction will lead to valid diagrams, it is tempting to perform this task with a computer.

Indeed, computer algebra systems like REDUCE can perform algebraic operations on mathematical expression, like addition, multiplication, differentiation, and integration. They provide a programming environment in which new procedures can be defined which can perform more complicated operations. REDUCE can handle various types of objects, e.g. mathematical expressions, arrays, and matrices. Special objects are lists of other objects, $\{A, B, C\}$. These objects can be lists themselves. An elementary interaction operator or a single Fourier component of a particular matrix element of the statistical operator can be represented by a list of four items, a 4-list, in the following way,

$\hat{V}_{r s}\left(\omega_{\alpha}\right) \rightarrow\left\{r, s, \omega_{\alpha}, V\right\}$,

$\hat{\rho}_{k l}\left(\omega_{\alpha}\right) \rightarrow\left\{k, l, \omega_{\alpha}, \rho\right\}$, 
where $V=-\mu_{r s} \cdot \mathscr{E}_{\alpha} / 2$ and $\rho$ are the values of the matrix elements. The first two items in each 4-list are the ketand bra-index of the elementary operator. These indices are integer numbers labelling the levels in ascending order. These are used to address the relevant molecular parameters from predefined matrices. The complete operators $V$ and $\rho$ are represented by lists of such 4-lists. E.g., the interaction operator relevant to the three-color grating experiment in a three level system as discussed in fig. 3 is

$V:=\left\{\left\{1,3, \omega_{1}, V_{1}\right\},\left\{3,1,-\omega_{2}, V_{2}\right\},\left\{1,2, \omega_{3}, V_{3}\right\}\right\}$.

Diagrams are also represented by lists which begin with the zero order density matrix element. A radiative propagation step is indicated by the key-word "BRA" or "KET" followed by the 4-list of the interaction operator involved. Feeding is indicated by the key-word "FEED" and the list $\{m, l\}$, where $m$ and $l$ are the indices of the upper and lower level. E.g., the diagram vii in fig. 3 is coded by the string \{START, 1, 1, 0, 1, KET, 3, 1, $-\omega_{2}, V_{2}$, BRA, 1, 3, $\omega_{1}, V_{1}$, FEED, 3, 1, BRA, 1, 2, $\left.\omega_{3}, V_{3}\right\}$.

The calculation of a susceptibility involves the following steps:

- Define the starting density operator. In most cases this is only a single matrix element, namely $\rho_{a a}^{(0)}=1$, represented by $\rho=\{\{1,1,0,1\}\}$.

- Define the interaction operator as in eq. (80).

- Specify a list of interactions to be considered in the perturbation scquence. E.g., VLIST: $=\{1,1,2,2,3\}$ specifies a fifth order calculation with the interactions $V_{1}$ and $V_{2}$ considered twice resulting in the Fourier component at $2 \omega_{1}-2 \omega_{2}+\omega_{3}$.

- Set switches that determine whether feeding and the rotating wave approximation are to be considered or not.

- call the SUSCEPTIBILITY procedure.

This procedure first generates a list of all permutations of VLIST. Then it propagates all initial density matrix elements according to all permuted time-ordered lists of interactions. In every step it is checked whether a braand/or ket-propagation is possible, and the resulting diagrams are added to the list for the next higher order of perturbation theory. This list is then checked for the occurrence of population terms, and if the switch for feeding is set, all diagrams that can be generated by feeding to lower levels are appended to the list. After all possible diagrams have been generated in this way, the corresponding contributions to the susceptibility are calculated according to the propagation rules (eqs. (41), (42), and (44)). The resulting expressions for each diagram can be printed. The value returned by the procedure is the sum of all terms after taking the limit $I_{a a}^{\prime} \rightarrow 0$. The procedure for the calculation of response functions works in complete analogy, except that the list of interactions is not permuted and the limit $\Gamma_{a a} \rightarrow 0$ is not taken. The returned value is then the response function for a particular time sequence of interactions, which may contain several diagrams.

As mentioned earlier, the contribution of a single diagram to the susceptibility can diverge if infinite lifetime is assumed for the ground state. Therefore, the limit $\Gamma_{a a} \rightarrow 0$ must be performed after the terms corresponding to a particular time sequence of interactions have been summed. However, care has to be exercised in using the relation between the decay rate constants and the feeding parameters, eq. (23), at the same time. As an example we discuss the three diagrams shown in fig. 6 which give the second-order contribution of frequency $\omega_{1}-\omega_{2}$ to

(i)

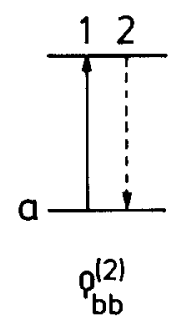

(ii)

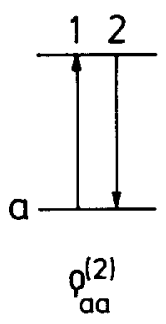

(iii)

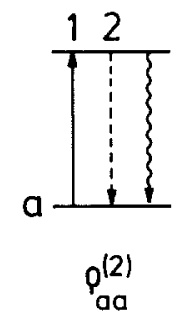

Fig. 6. Second-order diagrams describing the Fourier components at frequency $\omega_{1}-\omega_{2}$ of the populations in a two-level system. For discussion see text. 
the populations of a two-level system for the time ordering $V_{1}$ before $V_{2}$. The formulas for the corresponding steady-state density matrix elements are

$\rho_{b b}^{(2)}(\mathrm{i})=\frac{-A}{d-\mathrm{i} \Gamma_{b b}}$,

$\rho_{a a}^{(2)}(\mathrm{ii})=\frac{A}{d-\mathrm{i} \Gamma_{a a}}$,

$\rho_{a a}^{(2)}(\mathrm{iii})=\frac{\mathrm{i} \gamma_{b a} A}{\left(d-\mathrm{i} \Gamma_{b b}\right)\left(d-\mathrm{i} \Gamma_{a a}\right)}$,

with $d=\omega_{1}-\omega_{2}$ and

$A=\frac{1}{4 \hbar^{2}} \frac{\mu_{a b} \cdot \mathscr{E}_{1} \mu_{b a} \cdot \mathscr{E}_{2}}{\omega_{a b}+\omega_{1}-\mathrm{i} \Gamma_{a b}}$

Conservation of population requires that the sum of these three terms must vanish, so that

$\rho_{a a}^{(2)}(\mathrm{ii})+\rho_{a a}^{(2)}(\mathrm{iii})=-\rho_{b b}^{(2)}(\mathrm{i})$.

The two-level case is correctly treated by setting $\Gamma_{a a}=\Gamma_{b b}$ and formally neglecting feeding, $\gamma_{b a}=0$. This indeed fulfils eq. (85). In the general case, however, we have to add the contributions to the same matrix element and perform the limit $\Gamma_{a a} \rightarrow 0$. With $\Gamma_{b b}=\gamma_{b a}$ this gives still the correct result if $d \neq 0$. However, if we consider a dcterm taking the same frequency for both interactions, the expression for $\rho_{a a}^{(2)}$ is

$\rho_{a a}^{(2)}=-\mathrm{i} A \frac{\Gamma_{b b}-\gamma_{b a}}{\Gamma_{b b} \Gamma_{a a}}$

Applying $\Gamma_{b b}=\gamma_{b a}$ leads to $\rho_{a a}^{(2)}=0$ which is obviously wrong. Taking the limit $\Gamma_{a a} \rightarrow 0$ leads to divergence which is also wrong.

One way out of this dilemma would be to define all frequencies involved in the perturbation sequence to be different. The relations between the feeding parameters and the decay parameters can then be used and the limit of infinite lifetime for the ground state can be taken. The actual frequencies are set into the final expression. Such a procedure would, however, greatly restrict the flexibility of our approach. The other option is to regard the use of eq. (23) also as a limit and couple it to that for the ground state lifetime. For this purpose, eq. (23) was replaced by

$\sum_{k} \gamma_{m k}=\Gamma_{m m}-\Gamma_{a a}$

For the three-level case we could show that this ansatz avoids the divergence problems, but we have no general proof for an arbitrary number of levels. However, many REDUCE calculations on the basis of eq. (87) were performed for systems with up to six levels, and the answer was always correct.

\section{Summary}

The diagrammatic method for the perturbative calculation of non-linear optical susceptibilities has been reviewed and extended to include incoherent population transfer from higher to lower levels. The corresponding diagrams allow an easy visualization of the predominant contributions arising when certain input light frequencies are in resonance with optically allowed molecular transitions. The method was further extended to the direct calculation of molecular non-linear optical response functions. These describe the reaction of the molecular system to a sequence of ultrashort light pulses and are hence more appropriate for the description of time- 
domain experiments than susceptibilities. The algorithm for the generation of the complete set of diagrams arising from a particular experimental situation could be programmed within the computer-algebra system REDUCE. This program also generates the formulas for the susceptibility or the response function from the diagrams. Recently we have also successfully applied this method to the calculation of absorption and spontaneous or stimulated emission spectra, which are properly described by time correlation functions of matrix elements of the statistical error.

\section{Acknowledgement}

The author gratefully acknowledges generous support by Professor F.P. Schäfer, by the Deutsche Forschungsgemeinschaft through the Leibniz-Prize program and project SFB 93 (Photochemie mit Lasern), and by the Fonds der Chemischen Industrie.

\section{References}

[1] N. Bloembergen, Nonlinear optics (Benjamin, New York, 1965).

[2] P.N. Butcher, Nonlinear optical phenomena (Ohio State University Engineering Publications, Columbus, 1965).

[3] F. Ouellette and M.M. Denariez-Roberge, Can. J. Phys. 60 (1982) 877, 1477.

[4] B. Dick and R.M. Hochstrasser, Chem. Phys. 75 (1983) 133.

[5] B. Dick and R.M. Hochstrasser, J. Chem. Phys. 81 (1984) 2897.

[6] N. Bloembergen, H. Lotem and R.T. Lynch Jr., Indian J. Pure Appl. Phys. 16 (1978) 151.

[7] A. Yariv, Quantum electronics, 2nd Ed. (Wiley, New York, 1975) p. 557.

[8] J.F. Ward, Rev. Mod. Phys. 37 (1965) 1.

[9] C.J. Bordé, Compt. Rend. Acad. Sci. (Paris) B 282 (1976) 341.

[10] Ch. J. Bordé, J.L. Hall, C.V. Kunasz and D.G. Hummer, Phys. Rev. A 14 (1976) 236.

[11] J. Bordé and Ch. J. Bordé, Compt. Rend. Acad. Sci. (Paris) B 285 (1977) 287.

[12] J. Bordé and Ch. J. Bordé, J. Mol. Spectry. 78 (1979) 353.

[13] J.-L. Oudar and Y.R. Shen, Phys. Rev. A 22 (1980) 1141.

[14] S.Y. Yee, T.K. Gustafson, S.A.J. Druet and J.-P.E. Taran, Opt. Commun. 23 (1977) 1.

[15] T.K. Yee and T.K. Gustafson, Phys. Rev. A 18 (1978) 1597.

[16] S.A.J. Druet, B. Attal, T.K. Gustafson and J.P. Taran, Phys. Rev. A 18 (1978) 1529.

[17] Y.-J. Yan and S. Mukamel, J. Chem. Phys. 88 (1988) 5735.

[18] Y.-J. Yan and S. Mukamel, J. Chem. Phys. 89 (1988) 5160.

[19] Y.-J. Yan and S. Mukamel, J. Chem. Phys. 94 (1991) 179.

[20] B. Dick and R.M. Hochstrasser, J. Chem. Phys. 78 (1983) 3398.

[21] R.J. Carlson and J.C. Wright, Phys. Rev. A 40 (1989) 5092.

[22] B. Dick, H.P. Trommsdorff and R.M. Hochstrasser, in: Nonlinear optical properties of organic molecules and crystals, Vol. 2, eds. D.S. Chemla and J. Zyss (Academic Press, New York, 1987) pp. 159-212.

[23] For the newest version see: A.C. Hearn, REDUCE user's manual, version 3.4., Report CP 78, RAND, 1991 (Santa Monica, CA 90407-2138).

[24] J. Ducuing, in: Nonlinear optics, eds. P.C. Harper and B.S. Wherrett (Academic Press, London, 1977).

[25] J. Herrmann and B. Wilhelmi, Laser für ultrakurze Lichtimpulse (Physik Verlag, Weinheim, 1984).

[26] M. Schubert and B. Wilhelmi, Einführung in die nichtlineare Optik (Teubner, Leipzig, 1971).

[27] G.C. Baldwin, An introduction to nonlinear optics (Plenum Press, New York, 1969).

[28] D.C. Hanna, M.A. Yuratich and D. Cotter, Nonlınear optics of free atoms and molecules (Springer, Berlin, 1979).

[29] M.D. Levenson, Introduction to nonlinear laser spectroscopy (Acadernic Press, New York, 1982).

[30] B. Dick, Chem. Phys. 113 (1987) 131. 